\title{
Optical tolerance to rotation of trifocal toric IOLs as a function of the cylinder power
}

\begin{tabular}{|c|c|}
\hline Journal: & European Journal of Ophthalmology \\
\hline Manuscript ID & EJO-20-0297.R1 \\
\hline Manuscript Type: & Original Research Article \\
\hline $\begin{array}{r}\text { Date Submitted by the } \\
\text { Author: }\end{array}$ & $\mathrm{n} / \mathrm{a}$ \\
\hline Complete List of Authors: & $\begin{array}{l}\text { Ruiz-Alcocer, Javier; Complutense University of Madrid, Faculty of Optics } \\
\text { and Optometry } \\
\text { Lorente-Velázquez, Amalia; Complutense University of Madrid, Faculty of } \\
\text { Optics and Optometry } \\
\text { De Gracia, Pablo; Midwestern University - Downers Grove Campus, } \\
\text { Chicago College of Optometry } \\
\text { Madrid-Costa, David; Complutense University of Madrid, Faculty of } \\
\text { Optics and Optometry }\end{array}$ \\
\hline Keywords: & $\begin{array}{l}\text { IOLs < LENS / CATARACT, Optics/Refraction/Instruments < OPTICS / } \\
\text { REFRACTION / INSTRUMENTS, Intraoperative Complications of Cataract } \\
\text { Surgery < LENS / CATARACT, Corneal Topography < LENS / CATARACT, } \\
\text { IOL Calculation For Primary / Secondary IOL < LENS / CATARACT }\end{array}$ \\
\hline Abstract: & $\begin{array}{l}\text { Background: To assess the impact of } 5 \text {-degree and } 10 \text {-degree rotations } \\
\text { in the optical quality of a trifocal toric intraocular lens (IOL) with } \\
\text { different amounts of cylinder. } \\
\text { Methods: Two Physiol Toric IOL with } 1.5 \text { diopters (D) and 3.0D of } \\
\text { cylinder were analyzed in three different positions: centered, 5-degrees } \\
\text { and } 10 \text {-degrees rotated. The optical quality of the IOLs was evaluated } \\
\text { with the PMTF optical bench through specific perpendicular targets. The } \\
\text { analysis was performed by the through-focus modulation transfer } \\
\text { function (MTF) curves and the MTF corresponding to distance vision (0 D } \\
\text { of vergence). } \\
\text { Results: For a centered situation, the through-focus MTF curves of both } \\
\text { IOLs showed the classical three peaks corresponding to the powers of } \\
\text { the two principal meridians of the IOLs. When } 5 \text { and } 10 \text { degrees of } \\
\text { rotation were induced, the three peaks were attenuated in both cases. } \\
\text { The case with the IOL with } 3.0 D \text { of cylinder and } 10 \text { degrees of rotation } \\
\text { showed the worst optical quality and a significant loss of trifocality. The } \\
\text { MTF values obtained for distance vision also showed the worst optical } \\
\text { quality for the IOL with } 3.0 D \text { of cylinder and } 10 \text { degrees of rotation. } \\
\text { Conclusion: Rotations over } 5 \text { degrees decreased the optical quality of } \\
\text { trifocal toric IOLs, being this reduction moderate from } 5 \text { degrees to } 10 \\
\text { degrees for low levels of cylinder ( } \leq 1.5 D \text { ). For mid-high levels of cylinder } \\
\text { ( } \geq 3.0 D \text { ), rotations over } 5 \text { degrees cause a significant loss of optical } \\
\text { quality at all object distances. }\end{array}$ \\
\hline
\end{tabular}




\section{SCHOLARONE ${ }^{\text {W }}$ Manuscripts}




\section{ABSTRACT}

Background: To assess the impact of 5-degree and 10-degree rotations in the optical quality of a trifocal toric intraocular lens (IOL) with different amounts of cylinder.

Methods: Two Physiol Toric IOL with 1.5 diopters (D) and 3.0D of cylinder were analyzed in three different positions: centered, 5-degrees and 10-degrees rotated. The optical quality of the IOLs was evaluated with the PMTF optical bench through specific perpendicular targets. The analysis was performed by the through-focus modulation transfer function (MTF) curves and the MTF corresponding to distance vision ( $0 \mathrm{D}$ of vergence).

Results: For a centered situation, the through-focus MTF curves of both IOLs showed the classical three peaks corresponding to the powers of the two principal meridians of the IOLs. When 5 and 10 degrees of rotation were induced, the three peaks were attenuated in both cases. The case with the IOL with 3.0D of cylinder and 10 degrees of rotation showed the worst optical quality and a significant loss of trifocality. The MTF values obtained for distance vision also showed the worst optical quality for the IOL with 3.0D of cylinder and 10 degrees of rotation.

Conclusion: Rotations over 5 degrees decreased the optical quality of trifocal toric IOLs, being this reduction moderate from 5 degrees to 10 degrees for low levels of cylinder ( $\leq 1.5 \mathrm{D})$. For mid-high levels of cylinder ( $\geq 3.0 \mathrm{D})$, rotations over 5 degrees cause a significant loss of optical quality at all object distances. 


\section{INTRODUCTION}

Multifocal intraocular lenses (IOLs) were developed in order to increase depth of focus and offer spectacle independence to patients that undergo cataract surgeries. The working principle of these lenses was the creation of simultaneous retinal images, dividing the incoming light into two foci. ${ }^{1}$ Clinical results obtained with these bifocal designs showed a characteristic pattern with two peaks corresponding to near and far vision plus a gap in between for intermediate vision where optical quality rarely met the expectations of patients. ${ }^{2,3}$ Today, multiple trifocal IOLs are available in the market and clinical results in patients implanted with trifocals show three clear peaks of vision (far, intermediate, and near) the intermediate peak overcomes the mentioned limitation of bifocals. ${ }^{4,5}$

Some patients have significant amounts of corneal astigmatism: for example, cataract removal surgical procedures induce sometimes post-surgical astigmatism. And, this astigmatic values can alter the intended performance of IOLs. ${ }^{6}$ Therefore, trifocal toric designs have been developed to give satisfactory visual outcomes at all distances to astigmatic patients. Previous studies showed good clinical results with these lenses, ${ }^{7-9}$ the performance being, when they are properly implanted, similar to those obtained with their non-toric versions. ${ }^{9,10}$

Two main feats have to be properly attained in order to obtain a satisfactory toric IOL implantation and the subsequent good visual outcomes: accurate selection of the cylinder power and proper alignment of the IOL. ${ }^{11} \mathrm{~A}$ previous study has already assessed visual outcomes with bifocal toric and monofocal toric IOLs and has reported that misalignments have a greater impact in vision when bifocal designs are used. ${ }^{12}$ If proper centration is more critical when using spherical bifocal IOLs than when using monofocal IOLs, then, considering 
the importance of an accurate cylinder power calculation and a correct alignment of multifocal toric IOLs appears to be an interesting topic.

However, in classical clinical studies that examine misalignments of the IOLs, the potential degradation of the retinal image can be assessed only in one position, that is, the implanted IOL cannot be rotated towards different positions to assess the potential impact of these misalignments. Therefore, to assess the impact of several amounts of rotation by simulating these conditions in an optical bench becomes an extremely useful and noninvasive tool. In this study, we assess the performance of a widespread implanted trifocal toric IOL in terms of an optical quality metric (MTF) as a function of the amounts of prescribed cylinder in the IOL and of the amounts of rotation of the IOL. 


\section{MATERIALS AND METHODS}

\section{Intraocular lens designs studied}

The trifocal toric IOL analyzed was the hydrophilic acrylic (26\%) version of the FineVision Toric IOL (PhysIOL; Liège, Belgium). This IOL has a refractive index of 1.46 and incorporates both UV and blue-light filtration. The optical design is Biconvex, aspheric ($0.11 \mu \mathrm{m}$ Spherical Aberration for a $6.0 \mathrm{~mm}$ of pupil size), toric, trifocal, and diffractive. In the toric version, trifocality is obtained as in the non-toric version, that is, the lens combines two bifocal diffractive patterns for far/near and far/intermediate vision, respectively. ${ }^{13}$ The addition powers of the lens are: $+1.75 \mathrm{D}$ for intermediate and +3.50D for near vision. Additionally, the Physiol Toric presents an apodized design in which the step height decreases toward the periphery with increasing amount of light directed to distance vision. The lens shows a $360^{\circ}$ square edge. The overall diameter of the lens is $11.40 \mathrm{~mm}$ while the optic diameter is $6.00 \mathrm{~mm}$. The optical power of the lens goes from +6.0 to $+35.0 \mathrm{D}$ in $0.50 \mathrm{D}$ steps. For the present work, the sphere power of the lens was +20.0D. Among the cylinders available and as a representation of low and moderate-high astigmatism, ${ }^{14}$ two cylinder powers were analyzed: 1.5 and $3.00 \mathrm{D}$, respectively.

\section{Optical bench}

In order to assess the optical quality of optical systems such as IOLs, MTF measurements based on eye models are considered a standard and widespread method. ${ }^{15-20}$ Following the methodology employed in previous investigations, ${ }^{13,21,22}$ the image quality of each IOL was assessed with the PMTF optical bench (LAMBDA-X, Belgium) with Software version: 1.13.6. This device complies with International Standard Organization (ISO) 11979-2 
and 11979-9 requirements and allows MTF measurements at various frequencies, at different focal planes (through-focus) and for different pupil apertures.

\section{Optical quality assessment}

The pupil aperture chosen for the current work was $4.5 \mathrm{~mm}$ and the MTF was considered as the average modulation value which is the averaged values across all frequencies within the 0.0-to-100.0 cycles-per-millimetre range..$^{20,21,23}$ This value is proportional to the area under the MTF curve between 0.0 cycles $/ \mathrm{mm}$ and 120.0 cycles $/ \mathrm{mm}$.

In addition, through-focus MTF curves comprising 15 different focal points (steps of 0.5D) were calculated for a discrete spatial frequency of 50 cycles per millimetre.[14, 22] This spatial frequency could approximately correspond to an optotype for 0.5 Snellenequivalent VA in white light. The higher MTF value in the curve the better optical quality of the lens at this focal point.

\section{Impact of rotations}

In this work, the IOLs under study were analyzed for centered and rotated positions. For a centered position, the IOLs were measured with the meridians aligned with specific targets (vertical and horizontal edges) of the optical bench. These vertical and horizontal edges simulates the principal corneal meridians. Finally, for the analysis of the impact of misalignments and the potential degradation of the optical quality in the principal meridians, the IOLs were also rotated 5 and 10 degrees versus the aligned position and the optical quality was assessed at the specific targets. 


\section{RESULTS}

Figure 1 illustrates the through-focus MTF curves for the 1.5D cylinder power lens.

Figure 1.A shows the results of this lens in a centered position, while figure 1.B and 1.C show the results of the lens with 5 and 10 degrees of rotation versus the centered position, respectively. Figure 2 (2.A, 2.B and 2.C) present the results of the IOL with 3.0D of cylinder power. In figure 1 and figure 2, black lines represent the optical quality in one of the specific targets (edges) and the dotted grey line represent the optical quality in the perpendicular one. The space between the main peak values represents the amount of the cylinder of the lens.

Figure 1A (1.5D cylinder - centered) shows three peaks corresponding to far, intermediate and near distances of vision. Intermediate and near peaks of vision correspond to the addition powers of this design: 1.75 and 3.5D. At the same time, the lens presents a coherent difference of 1.5D between each corresponding pair of peaks (far to far, intermediate to intermediate and near to near) that corresponds to the cylinder power of the IOL.

In figure 1.B, when the 1.5D cylinder lens is rotated 5 degrees, the through-focus curve shows a degradation of the optical quality at the three peaks of vision by up to a $50 \%$. The larger amount of degradation occurs for the far distance peak ( $\approx 50 \%$ decrease) while the intermediate $(\approx 20 \%$ decrease) and near peaks showed ( $\approx 20 \%$ decrease) slightly lower values if compared to the centered situation (figure 1.A).

The results for 10 degrees of rotation are presented in figure 1.C. In this case, the overall optical quality is worse ( $\approx 58 \%$ decrease) if compared to the centered position 
(figure 1.A) and the overall optical quality show a moderate increase (additional $\approx 10 \%$ ) in the degradation of the image if compared to the misaligned position of $\mathbf{5}$ degrees (figure 1.B). The three peaks show similar MTF values if compared to the 5 degrees situation (figure 1.B). Then, the curves presented in figure 1 indicate an overall worsening of the optical quality for rotations of 5 degrees that remain constant up to 10 degrees.

Figure 2 shows the trough-focus analysis for the IOL with 3.0D of cylinder (moderate-high). The dotted grey line in this figure confirm the optical power difference between both meridians (3.0D of cylinder power) for each pair of peaks. In this case, the centered position (figure 2.A) also shows the three peaks placed at far, 1.75 and 3.5D, with nearly the same values than in the low cylinder case (figure 1.A).

When the lens is rotated 5 degrees (figure 2.B), the overall optical quality decreases in a similar way than the low cylinder case (figure 1.B). If compared to the centered position, there is a significant worsening of the optical quality for the far distance peak ( $\approx$ $55 \%$ decrease) but only a slight degradation for the intermediate and near peaks ( $\approx 9 \%$ decrease).

The rotation of 10 degrees (figure 2.C) in the moderate-high cylinder (3.0D) IOL causes a strong deterioration of the optical quality at all vergences with significant low values if compared to the centred situation (figure 2.A). Hence, figure 2 shows that for this cylinder power (3.0D), the optical quality showed and abrupt deterioration from 5 to 10 degrees.

So as to analyze the degradation of the image quality for distance vision when the trifocal toric $10 L$ is misaligned (5 and 10 degrees), figure 3 show the respective MTF curves. Figure 3.A and 3.B present the MTF curve of the 1.5D and 3.0D cylinder power IOLs, 
respectively. At the same time, table 1 summarizes the average modulation values (which are proportional to the area under the MTF curve (the higher the area the better optical quality) between 0.0 cycles $/ \mathrm{mm}$ and 120.0 cycles $/ \mathrm{mm}$ ) for the far (distance) focal point in all the situations abovementioned. In table 1, the percentage of energy loosed for misaligned positions related to the centered one is also presented.

Figure 3.A (1.5D cylinder power) and table 1 show results in which the MTF at far distance is better for a centered situation. When the IOL is rotated 5 degrees, the optical quality decreases by $25 \%$ with respect to the centered situation. Similarly, with 10 degrees of rotation the optical quality decreases by $30 \%$ with respect the centered position. Therefore, the degradation of the optical quality for distance vision is similar between 5 and 10 degrees for the low cylinder IOL.

Figure 3.B (3.0D cylinder power) and table 1 show that the best MTF value is reached also for the centered situation. In this case, 5 degrees of rotation induces an optical degradation for distance vision of $28 \%$ with respect the centered situation. This degradation is also similar to that obtained for the low cylinder IOL. However, greater differences can be found for 10 degrees of rotation. Figure 3.B and table 1 shows that the MTF value decreases by $46 \%$ with respect to the centered position (and $18 \%$ with respect 5 degrees of rotation) for this extreme situation. 


\section{DISCUSSION}

In the present study, the impact of two different degrees of rotation in a trifocal toric IOL with two different cylinder powers (low and moderate-high) is assessed. By comparing these situations, tolerance to IOL rotation was also assessed. The results of the present study show that the amount of cylinder does not have an impact on the optical quality of the trifocal toric IOL when it is properly aligned. However, the amount of cylinder is crucial when the $\mathrm{IOL}$ is rotated because there is a strong positive correlation between the amount of degradation of the image and the amount of the cylinder power.

The analysis of the through-focus for the IOL with 1.5D of cylinder (low cylinder) in centered position is showed in figure $1 \mathrm{~A}$. As mentioned in the results, this figure shows the characteristic three peaks of vision of trifocal IOLs. In this case, the peaks are placed at far (0.0D), 1.75 and 3.5D of addition. These three peaks have been related to three zones with improved visual quality of patients implanted with this IOL design. ${ }^{9,} 24$

When the lens is rotated 5 degrees, the through-focus analysis (figure 1.B) shows a degradation of the optical quality by decreasing the three peaks of the original MTF. Despite the overall worsening of the optical quality of the IOL, the most significant degradation occurs for the far distance peak compared to the centered situation. When the lens was tilted 10 degrees (figure 1.C), the three peaks showed a similar degradation if compared to the misaligned position of 5 degrees (figure 1.B). These results suggest that rotations of 5 degrees reduce optical quality post-surgery and this decrease could have an impact on the visual quality of patients. Besides, results also show that this drop remains constant up to 10 degrees of rotation for lenses with 1.5D of cylinder power.

For the moderate-high (3.0D) cylinder IOL, the trough-focus analysis showed that the centered position (figure 2.A) generated the same three peaks of vision than the low 
cylinder IOL. When the lens was rotated 5 degrees (figure 2.B), the optical quality decreases in a similar way than the low cylinder case. By contrast, the rotation of 10 degrees (figure 2.C) for the higher cylinder lens induced a significant degradation of the optical quality at all vergences. The figure shows a trough-focus curve with significant low values if compared to the centred situation and a significant decrease on the visual quality at all distances should be expected for this amount of rotation. In contrast to the cylinder of 1.5D, where the optical behaviour remained constant from 5 to 10 degrees of rotation, for 3.0D of cylinder the optical quality decreased steeply from 5 to 10 degrees.

As it can been observed in the through focus curves, the significant worsening of the optical quality in the far distance peak when the lenses are rotated lead into a deeper analysis of this focal point through the MTF and the modulation averaged values. Therefore, in order to calculate the impact of potential rotations on the optical quality at the far distance focus, the abovementioned situations were studied in detail this crucial focal point for the IOLs under study.

For the case of the trifocal toric IOL with low cylinder power (1.5D), figure 3.A and table 1 show logical results in which the MTF at far distance is better for a centered situation. When the $\mathrm{IOL}$ is rotated 5 and 10 degrees, the optical quality decreases by $25 \%$ and $30 \%$, respectively. Despite the 10 degrees of rotation being the worst situation, it is worth noticing how the loss of optical quality between 5 and 10 degrees of rotation is barely a $5 \%$. Therefore, it could be suggested that tolerance to rotation with low cylinder power IOLs is up to 10 degrees. However, it is important to note that a significantly better optical quality at distance vision is achieved when the IOL is perfectly centered.

Observing the results of the moderate-high cylinder (3.0D) IOL (figure 3.B and table 1), there are some differences if compared to the low cylinder lens. In this case, the best 
MTF value is reached also for the centered situation and its value is similar to the IOL with low cylinder. Besides, 5 degrees of rotation induced an optical degradation of $28 \%$ with respect the centered situation, which is a similar decrease if compared to the low cylinder case. However, figure 3.B and table 1 showed a steep deterioration for 10 degrees of rotation ( $46 \%$ with respect to the centered position). Then, it could be said that for moderate-high cylinder trifocal IOLs, rotations over 5 degrees could have a significant impact on distance vision.

As it was reported in previous investigations one degree of misalignment causes a loss of approximately $3 \%$ of the effective cylinder power, and the entire toric effect is lost in cases with $30^{\circ}$ of misalignment. ${ }^{25,26}$ In relation to this and to the impact of rotations on visual quality, a previous investigation analysed visual outcomes at distance vision with multifocal toric and monofocal toric IOLs. ${ }^{12}$ The authors reported that misalignments have a greater impact in vision with multifocal designs. ${ }^{12}$ In this study, authors studied a bifocal IOL, hence, it could be suggested that a proper implantation for trifocal toric lenses would also be crucial.

The results of the present study showed a certain amount of tolerance to rotations (or constant decrease of image quality) for low cylinders. However, this "tolerance" should be taken with caution because even 5 degrees of rotation deteriorates the image quality of both IOLs (table 1). To correlate the optical quality and its impact on visual quality, Felipe et al.[28] reported that a reduction over $15 \%$ in the MTF could affect visual acuity. Therefore, the present study suggest that rotations over 5 degrees on trifocal toric IOLs could have an impact on the optical quality of patients. However, direct comparisons between both studies should be made with caution because Felipe et al. ${ }^{27}$ did not considered trifocal or trifocal 
toric IOLs. Then, they could not estimate the optical quality as a function of the parameters analyzed in the present study, that is, the degree of rotation and amount of cylinder.

The objective assessment of the impact of different degrees of rotations in the same patient is not feasible under any circumstances. Therefore, an optical bench approach becomes very useful. That being said, the results in this study do not represent real clinical situations and present limitations related to the impact on patient's visual quality. Still the procedure used represents a useful and non-invasive method for the analysis of multiple levels of astigmatic power and rotation values on equal footing.

In conclusion, even if toric IOLs show a positive trend in terms of stability, the results of the current study show that rotations over 5 degrees in trifocal toric IOL with $\geq 3.0 \mathrm{D}$ of cylinder cause a significant loss of optical quality that may cause a significant loss of visual quality at all distances of vision. Then, pre and intraoperative techniques are crucial for monitoring proper centration and rotation of trifocal toric IOL implantations, especially in IOLs with moderate-high cylinders. 


\section{Declaration of Conflicting Interests}

The authors declare that there is no conflict of interest.

\section{Funding}

This research received no specific grant from any funding agency in the public, commercial, or not-for-profit sectors. 


\section{REFERENCES}

1. Montés-Micó R, España E, Bueno I, et al. Visual performance with multifocal intraocular lens: mesopic contrast sensitivity under distance and near conditions. Ophthalmology 2004; 111:85-96.

2. Alfonso JF, Fernández-Vega L, Puchades C, et al. Intermediate visual function with different multifocal intraocular lens models. J Cataract Refract Surg 2010; 36:733-739.

3. Pepose JS, Wang D, Altmann GE. Comparison of through-focus image sharpness across five presbyopia-correcting intraocular lenses. Am J Ophthalmol 2012; 154:20-28.

4. Xu Z, Cao D, Chen X, et al. Comparison of clinical performance between trifocal and bifocal intraocular lenses: A meta-analysis. PLOS ONE 2017; 12:e0186522 https://doi.org/10.1371/journal.pone.0186522

5. Jonker SM, Bauer NJ, Makhotkina NY, et al. Comparison of a trifocal intraocular lens with a +3.0 D bifocal IOL: results of a prospective randomized clinical trial. J Cataract Refract Surg 2015; 41:1631-1640.

6. Piovella M, Colonval S, Kapp A, et al. Patient outcomes following implantation with a trifocal toric IOL: twelve-month prospective multicentre study. Eye (Lond) 2019; 33:144-153.

7. Gundersen KG and Potvin R. Comparison of visual outcomes after implantation of diffractive trifocal toric intraocular lens and a diffractive apodized bifocal toric intraocular lens. Clin Ophthalmol 2016; 10:455-461. 8. Mojzis P, Majerova K, Plaza-Puche AB, et al. Visual outcomes of a new toric trifocal diffractive intraocular lens. J Cataract Refract Surg 2015; 41:2695-2706. 9. Rementería-Capelo LA, Contreras I, García-Pérez JL, et al. Visual quality and patient satisfaction with a trifocal intraocular lens and its new toric version. J Cataract Refract 
Surg 2019; 45:1584-1590.

10. Poyales F and Garzon N. Comparison of 3-month visual outcomes of a spherical and a toric trifocal intraocular lens. J Cataract Refract Surg 2019; 45:135-145.

11. Zhou F, Jiang W, Lin Z, et al. Comparative meta-analysis of toric intraocular lens alignment accuracy in cataract patients: Image-guided system versus manual marking. $J$ Cataract Refract Surg 2019; 45:1340-1345.

12. Garzón N, Poyales F, de Zárate BO, et al. Evaluation of rotation and visual outcomes after implantation of monofocal and multifocal toric intraocular lenses. J Refract Surg 2015; 31:90-97.

13. Gatinel D and Loicq J. Clinically Relevant Optical Properties of Bifocal, Trifocal, and Extended Depth of Focus Intraocular Lenses. J Refract Surg 2016; 32:273-280.

14. Emesz M, Dexl AK, Krall EM, et al. Randomized controlled clinical trial to evaluate different intraocular lenses for the surgical compensation of low to moderate-to-high regular corneal astigmatism during cataract surgery. J Cataract Refract Surg 2015;

41:2683-2694.

15. Rawer R, Stork W, Spraul CW, et al. Imaging quality of intraocular lenses. J Cataract Refract Surg 2005; 31:1618-1631.

16. Kawamorita T and Uozato $\mathrm{H}$. Modulation transfer function and pupil size in multifocal and monofocal intraocular lenses in vitro. J Cataract Refract Surg 2005; 31:2379-2385. 17. Artigas JM, Menezo JL, Peris C, et al. Image quality with multifocl intraocular lenses and the effect of pupil size; comparison of refractive and hybrid refractive-diffractive designs. J Cataract Refract Surg 2007; 33:2111-2117.

18. Altmann GE, Nichamin LD, Lane SS, et al. Optical performance of 3 intraocular lens designs in the presence of decentration. J Cataract Refract Surg 2005; 31:574-585. 
19. Lorente A, Pons AM, Malo J, et al. Standard criterion for fluctuations of modulation transfer function in the human eye: application to disposable contact lenses. Ophthalmic Physiol Opt 1997; 17:267-272.

20. Artigas JM, Peris C, Felipe A, et al. Modulation transfer function: rigid versus foldable phakic intraocular lenses. J Cataract Refract Surg 2009; 35:747-752.

21. Ruiz-Alcocer J, Madrid-Costa D, García-Lázaro S, et al. Optical performance of two new trifocal intraocular lenses: through-focus modulation transfer function and influence of pupil size. Clin Exp Ophthalmol 2014; 42:271-276.

22. Ortiz C, Esteve-Taboada JJ, Belda-Salmerón L, et al. Effect of Decentration on the Optical Quality of Two Intraocular Lenses. Optom Vis Sci 2016; 93:1552-1559.

23. Madrid-Costa D, Ruiz-Alcocer J, Ferrer-Blasco T, et al. Optical quality differences between three multifocal intraocular lenses: bifocal low add, bifocal moderate add, and trifocal. J Refract Surg 2013; 29:749-754.

24. Cochener B, Vryghem J, Rozot $P$, et al. Clinical outcomes with a trifocal intraocular lens: a multicenter study. J Refract Surg 2014; 30:762-768.

25. Ma JJ and Tseng SS. Simple method for accurate alignment in toric phakic and aphakic intraocular lens implantation. J Cataract Refract Surg 2008; 34:1631-1636.

26. Till JS, Yoder PR Jr, Wilcox TK, et al. Toric intraocular lens implantation: 100 consecutive cases. J Cataract Refract Surg 2002; 28:295-301.

27. Felipe A, Pastor F, Artigas JM, et al. Correlation between optics quality of multifocal intraocular lenses and visual acuity; tolerance to modulation transfer function decay. $J$ Cataract Refract Surg 2010; 36:557-562. 


\section{FIGURE LEGENDS}

Figure 1. Through-focus MTF curves for the Physiol Toric intraocular lens with 1.5 diopters of cylinder (low cylinder) for: centered position (A), 5 degrees of rotation (B) and 10 degrees of rotation (C). Black and dotted lines represent the results obtained for each of the two principal meridians of the IOL. The curves were calculated for a spatial frequency of 50 cycles per millimetre (cycles/mm). This spatial frequency could approximately correspond to an optotype for 0.5 Snellen-equivalent VA in white light. The higher MTF value in the curve the better optical quality of the lens at this focal point.

Figure 2. Through-focus MTF curves for the Physiol Toric intraocular lens with 3.0 diopters of cylinder (moderate-high cylinder) for: centered position (A), 5 degrees of rotation (B) and 10 degrees of rotation (C). Black and dotted lines represent the results obtained for each of the two principal meridians of the IOL. The curves were calculated for a spatial frequency of 50 cycles per millimetre (cycles $/ \mathrm{mm}$ ). This spatial frequency could approximately correspond to an optotype for 0.5 Snellen-equivalent VA in white light. The higher MTF value in the curve the better optical quality of the lens at this focal point.

Figure 3. MTF curves for the three different situations (centered, 5 degrees and 10 degrees of rotation) at the far distance focal point ( 0.0 diopters vergence) for the Physiol Toric intraocular lens with 1.5 diopters of cylinder (low cylinder) (A) and 3.0 diopters of cylinder (moderate-high cylinder) (B). 
Table 1. Average modulation values at the far (distance) focal point for of the intraocular lens and the cylinder powers under study for $4.5 \mathrm{~mm}$ of aperture.

\section{Cylinder power (D)}

1.5 D $\quad 3.0 \mathrm{D}$
Centered
38.58
37.74

\begin{tabular}{lcc}
\hline $\mathbf{5}$ degrees & 28.89 & 27.07 \\
& & \\
\hline loss energy & $25 \%$ & $28 \%$ \\
vs centered & & \\
\hline 10 degrees & 27.02 & 20.32 \\
& & \\
\hline loss energy & & \\
vs centered & $30 \%$ & 46 \\
\hline
\end{tabular}



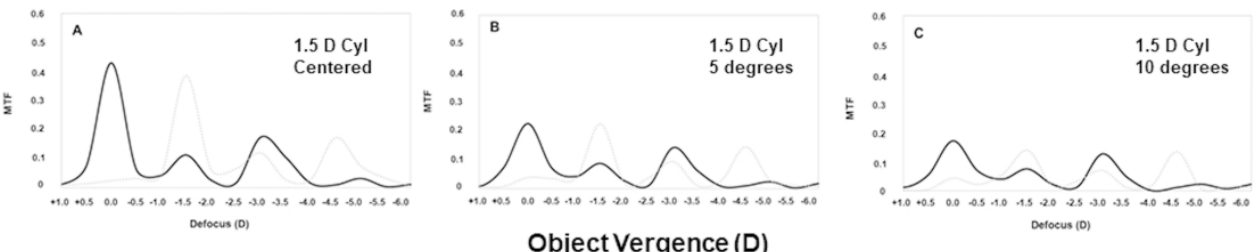

Through-focus MTF curves for the Physiol Toric intraocular lens with 1.5 diopters of cylinder (low cylinder) for: centered position (A), 5 degrees of rotation (B) and 10 degrees of rotation (C). Black and dotted lines represent the results obtained for each of the two principal meridians of the IOL. The curves were calculated for a spatial frequency of 50 cycles per millimetre (cycles/mm). This spatial frequency could approximately correspond to an optotype for 0.5 Snellen-equivalent VA in white light. The higher MTF value in the curve the better optical quality of the lens at this focal point.

$254 \times 59 \mathrm{~mm}(1200 \times 1200$ DPI $)$ 

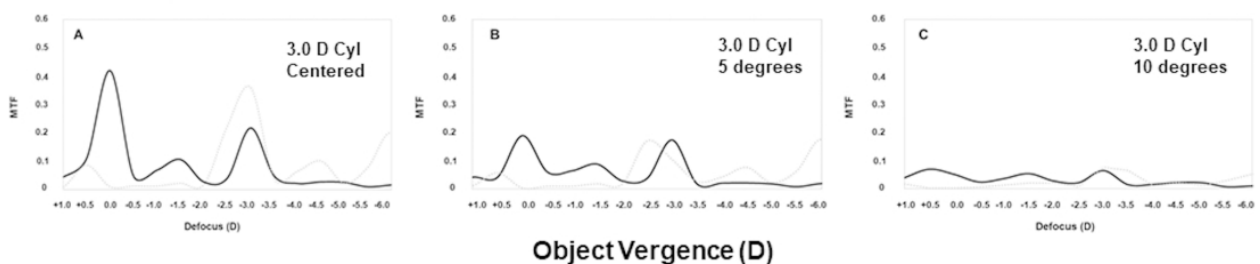

Through-focus MTF curves for the Physiol Toric intraocular lens with 3.0 diopters of cylinder (moderate-high cylinder) for: centered position (A), 5 degrees of rotation (B) and 10 degrees of rotation (C). Black and dotted lines represent the results obtained for each of the two principal meridians of the IOL. The curves were calculated for a spatial frequency of 50 cycles per millimetre (cycles $/ \mathrm{mm}$ ). This spatial frequency could approximately correspond to an optotype for 0.5 Snellen-equivalent VA in white light. The higher MTF value in the curve the better optical quality of the lens at this focal point.

$254 \times 60 \mathrm{~mm}(1200 \times 1200 \mathrm{DPI})$ 

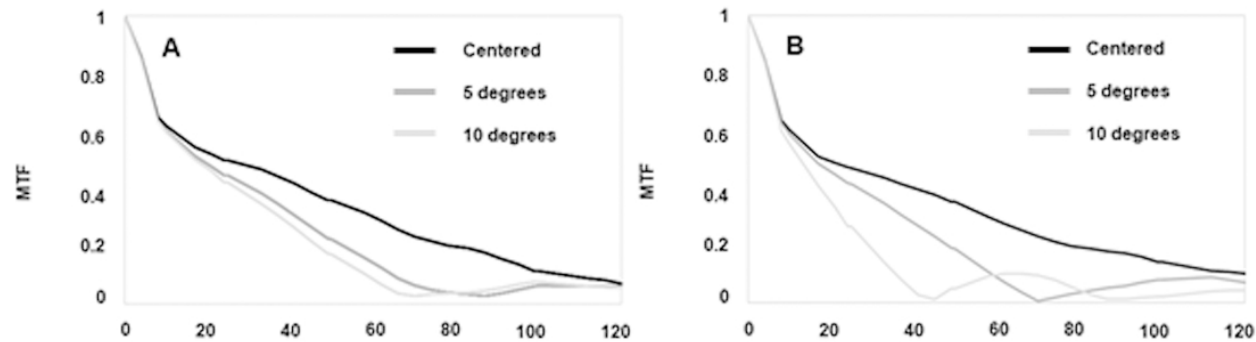

Spatial frequency (cycles $/ \mathrm{mm}$ )

MTF curves for the three different situations (centered, 5 degrees and 10 degrees of rotation) at the far distance focal point (0.0 diopters vergence) for the Physiol Toric intraocular lens with 1.5 diopters of cylinder (low cylinder) (A) and 3.0 diopters of cylinder (moderate-high cylinder) (B).

$157 \times 64 \mathrm{~mm}(1200 \times 1200 \mathrm{DPI})$ 